Highlights

Advanced tools and resources are available to silence or overexpress Anopheles genes

Precise genome editing is broadly applicable to multiple Anopheles species

Proof of principle for Anopheles population suppression and modification are successful in laboratory settings 


\title{
Opening the toolkit for genetic analysis and control of Anopheles mosquito vectors
}

\author{
Adriana Adolfia and Gareth John Lycett ${ }^{\mathrm{b}}$
}

\author{
a Department of Microbiology and Molecular Genetics, School of Medicine, University of \\ California, Irvine, CA 92697-4500, USA \\ b Vector Biology Department, Liverpool School of Tropical Medicine, Liverpool, L3 5QA, UK
}

\begin{abstract}
Anopheles is the only genus of mosquitoes that transmit human malaria and consequently the focus of large scale genome and transcriptome-wide association studies. Genetic tools to define the function of the candidate genes arising from these analyses are vital. Moreover, genome editing offers the potential to modify Anopheles population structure at local and global scale to provide complementary tools towards the ultimate goal of malaria elimination. Major breakthroughs in Anopheles genetic analysis came with the development of germline transformation and RNA interference technology. Yet, the field has been revolutionized again by precise genome editing now possible through site-specific nucleases. Here we review the components of the current genetic toolkit available to study Anopheles, focusing particularly on how these technical advances are used to gain insight into malaria transmission and the design of genetic methods to control Anopheles vectors.
\end{abstract}

\section{Introduction}

Malaria, transmitted solely by Anopheles spp. mosquitoes, remains the most important global vector borne disease and a priority target for control, local eradication and global elimination. Despite the halving of global malaria deaths between 2000-2015, mainly due to enormous programs that successfully targeted Anopheles vectors with insecticide treated nets, 445,000 people still die annually from the disease and some indicators point to a stalling of progress [1]. There is therefore an urgent need to tackle malaria transmission using all the methods available, including improved insecticide, vaccine and drug development. To complement these public health approaches to disease control, advances in genetic methods have promised 'realizable' tools that will bolster the fight against Anopheles mosquitoes.

Large scale comparative sequencing projects are currently used to identify genes that potentially impact malaria transmission [2]. Understanding the physiological function of these candidate genes requires techniques that silence, mutate or overexpress them in vivo to characterize the resultant alteration in phenotype. Here we will review these genetic tools (summarised in Table1), illustrated with recent insights into how they may be used to study the mosquito biology influencing vectorial capacity, including the latest advances that are directed towards Anopheles population control. 


\section{Functional genetic analysis}

\section{RNA interference (RNAi)}

Main current use: rapid validation of the effects of gene knockdown on vector phenotype

Perhaps the most widely used method for genetic analysis in Anopheles mosquitoes has been RNAi. The technique can be highly gene specific, since it relies on near complete homology to target specific mRNAs for degradation (reviewed in [3]). The technique is based on the natural cellular response to the detection of double stranded (ds) RNA. After introducing synthetic dsRNA homologous to a target gene, the cellular machinery processes this template to direct cleavage of complementary mRNA, thereby reducing the encoded protein level over time (Fig $1 \mathrm{~A}$ ).

A recent extensive example of this approach involved silencing Anopheles innate immunity genes using a phenotype readout of prevalence and intensity of Plasmodium infection [4]. These experiments demonstrated that the antagonistic and agonistic effectors $[5,6]$ of Plasmodium development are differentially expressed in alternative Anopheles species in response to the intensity of parasite burden. This robust technique has also been applied to many other diverse aspects of Anopheles biology ranging from insecticide resistance [7] to heart rate [8]. Although most commonly used in adults, the technique can also be successfully applied to embryos [9] to study developmental gene function and, with some difficulty, to pupae [10]. Larvae do not survive the injection process well, and techniques have been recently modified to silence larval genes by feeding chitosan/dsRNA nanoparticles or bacteria/yeast expressing dsRNAs [11].

Overall, the technique provides rapid assessment of loss of gene function phenotypes, however in anophelines not all genes and/or tissues are targeted efficiently [12] and so care is needed to monitor protein (or mRNA) turnover. In addition, off-target silencing has the potential to complicate analysis, but has not been extensively studied in anophelines [3]. Nevertheless, the major advantage of this approach is that the effect of gene silencing can be monitored stage-specifically, which can often be more difficult to achieve with other methods.

By targeting vital genes, RNAi may play a role in anopheline and malaria control. Perhaps the closest to translation are RNAi-based larvicides delivered as dried pellets to defined larval breeding sources to provide Anopheles specific killing with limited toxicity to other organisms [11].

\section{Transposable elements}

Main current use: assaying phenotypes produced by tissue specific and ectopic expression of transgenes, promoter trapping, creating docking lines for comparative analysis

The RNAi methodology offers a one stage or one generation approach to loss of function analysis, and so needs to be continuously repeated to provide experimental material. By modification of germline chromosomes, stable mosquito lines are generated that inherit loss or gain of gene function phenotypes. Such transgenic anopheline lines were firstly created using processes adopted from Drosophila methodologies. These included embryo microinjection targeted to developing germline cells, permissive transposable element transformation vectors (Minos and PiggyBac) (Fig 1B) that catalyse integration of themselves (and linked DNA cargoes) into germline chromosomes, and promoter sequences to drive expression of selectable marker genes to visually detect the rare transgenic progeny $[13,14]$. Using these transposon-based methods and a range of cloned tissue-specific promoters [15] to regulate transgene expression, stable Anopheles mosquito lines can be generated that ectopically express endogenous/foreign genes or dsRNAs to examine phenotypic consequences of increased or 
decreased expression respectively. For example, as discussed in greater detail below, Plasmodium refractory strains of Anopheles have been developed through midgut expression of a number of different gene families [16].

The integration sites of transposons are nearly-random in the genome, often leading to variability in expression between lines carrying the same transgene in different genomic locations (position effect), and to insertional mutagenesis if transposon integration disrupts a coding sequence. The end result is that multiple lines need to be generated to draw reliable conclusions on the phenotype.

Although their use is likely to be superseded by the flexibility of site-specific approaches, the random nature of transposon insertion makes them particularly useful for unbiased screening techniques such as promoter trapping, whereby DNA regulatory regions can be identified following the insertion of a promoter-less fluorescent reporter gene in their vicinity [17]. Transposon integration can also be used to screen for genomic sites that produce limited fitness cost and consistent transgene expression [18], suitable for docking lines described in the following section.

\section{Site directed integration}

Main current use: comparative functional analysis

To normalise position effect variation of expression, docking lines have been created that direct the insertion of genes into 'tagged' genomic sites through the site-directed recombinase phic31 [19]. This is achieved by including a recombinase docking (attP) site in the integrated transgene to create a stable line tagged at a defined locus. Alternative transgenes can then be inserted into this locus by including a donor (attB) site in the new transformation vectors and a source of PhiC31 enzyme to catalyse the recombination reaction (Fig $1 \mathrm{Ci}$ ). Each line should express the alternative transgenes at similar temporal and spatial patterns and levels allowing robust comparison of relative gene function.

The phiC31 recombinase can also be used to precisely exchange transgenes into defined genomic sites (Fig 1Cii) by placing an attP site on either side of the docking transgene [18]. New DNA constructs carrying flanking attB sequences will then swop into these sites by recombination to seamlessly replace the original transgene [20].

A number of docking anopheline lines are now available and further refinements are discussed in [21]. This review also introduces a flow cytometry method (COPAS ${ }^{\mathrm{TM}}$ ) which allows rapid fluorescence screening of live mosquito larvae that speeds the process of generating and characterising transgenic lines.

\section{Bipartite expression systems}

Main current use: to study toxic genes, comparative functional analysis

The scope of Drosophila genetic analysis has been based largely on the use of bipartite expression systems. By separating the transgene under analysis (responder) from the regulatory regions controlling its expression (driver) in distinct transgenic lines, genes causing extreme fitness or developmental phenotypes can be examined in the progeny of driver and responder line crosses (Fig 1D). As well as examining toxic genes, bipartite systems also provide a flexible platform for comparative tissue specific expression analysis whereby different effector genes can be assayed in multiple expression patterns simply by crossing appropriate responder and driver lines.

Different bipartite systems have been utilized in Anopheles. The tTA/tetO system has been developed for gut [22] and flight muscle [23] specific expression in An. stephensi. Similarly, the GAL4/UAS has been used for enhancer trapping in An. stephensi [17], and ubiquitous [18] and gut specific expression [24] in 
An. gambiae. Finally, the Q system has been exploited to characterise An. gambiae olfactory receptor neurons [25].

In the latter case, piggyBac insertions created driver lines carrying the An. gambiae odorant receptor coreceptor (orco) promoter controlling QF2 transcription activator expression and responder lines carrying QF2 binding sites (QUAS) upstream of a membrane-localised green fluorescent protein (mGFP) gene. In the progeny of driver and responder crosses, the mGFP localisation recapitulated the endogenous orco expression pattern, and hence fluorescently tagged all neurons bearing olfactory receptors. In this way olfactory innervations that may be involved in host recognition were exquisitely mapped. The bipartite system generated opens one way to express reporter genes that can monitor real time activity in discreet neuronal families to define the olfactory 'code' of odour recognition during host seeking. Moreover, by adoption of the many other reporter based approaches used in Drosophila, it may be possible to modulate and disrupt host-seeking behaviour [25].

\section{Site specific nucleases}

Main current uses: site specific genome editing, gene knockout and knockin

The advent of relatively flexible and powerful genome editing tools based on a range of site-specific nucleases offers the potential for a vast array of precise genome modifications which is revolutionizing genetic analysis in Anopheles, as in other model and non-model organisms [26]. They all rely on site specific endonuclease cleavage of germline DNA that is repaired in the cell by either i) 'imperfect' non homologous end joining (NHEJ) that most often creates frameshift mutations, or ii) homology directed repair (HDR) whereby homologous sequences are used as a recombination template to repair the damage. HDR can be exploited by providing transgenes flanked by homologous sections around the known cut site, so that during repair the transgene cargo is copied into a precisely defined location. Although not published extensively yet in Anopheles, it is relatively straightforward to mutate or insert a DNA cargo into virtually any genomic location desired, particularly in the case of Cas 9 modifications as described and illustrated in Fig 1E.

Such site specific genome editing has been performed in anophelines using homing nucleases [27], transcription activator-like effector nucleases (TALENs)[28-30], and Cas9 nucleases [20,31-33]. With homing endonucleases and TALENs, specific genomic sequences targeted for cleavage are recognized directly by the nuclease, and so altering the target site can be a fairly intensive protein/DNA engineering process. Nevertheless, a homing endonuclease was the first used in Anopheles for site specific modification [27] (see below), and more recently TALENs were created for both gain and loss of function analysis of the TEP1 complement-like protein in An. gambiae to examine its role in Plasmodium killing $[29,30]$. A TALEN kynurenine 3-monooxygenase gene knockout in An. stephensi [28] also answered a long-standing question on the role of mosquito-derived xanthurenic acid in stimulating Plasmodium development in the mosquito midgut.

The CRISPR/Cas9 methods are generally much simpler to perform, since the Cas9 endonuclease is directed to a specific DNA locus site by a unique, complementary short guide RNA which can be rapidly designed and synthesised. Highly efficient CRISPR/Cas9-mediated NHEJ mutagenesis of visible phenotypic markers [31] has been demonstrated in multiple Anopheles species, as well as efficient HDR in An. stephensi [32] and An. gambiae [20], supporting the broad potential applications across the genus. With continual refinements in targeting efficiency, CRISPR/Cas9-based analysis thus seems likely to dominate genome modification approaches for the foreseeable future.

Most of the recent applications of CRISPR/Cas9 in anophelines have been in the field of gene drives and will be discussed in more detail below. However, the potential applications of CRISPR/Cas9 in functional 
genetic analysis of anophelines is very broad: base pair changes to introduce point mutations, insertion into coding regions to create gene knockouts, substitution of genes or fluorescent protein tagging, chromosome rearrangement, regulation of gene expression, and chromatin modification. The above list of potential applications is far from exhaustive and further developments will undoubtedly broaden this portfolio as technological improvements increase control, efficiency and specificity.

As neurobiological examples, it would be relatively straightforward to target different classes of sensory neurons (e.g. Fig 2 - Anopheles chordotonal organs) to co-express bipartite drivers with specific neuronal genes by Cas9-mediated HDR. By appropriate design and fusion with a 2A self-cleaving peptide [34], the driver coding sequence could be inserted into a known neuronal specific gene to achieve bicistronic expression regulated by the native promoter. Co-translational cleavage would be expected to produce equimolar amounts of the two proteins in active forms that would create a neuronal driver suitable to express a variety of reporters. These may include fluorescent tags for anatomical mapping [25], genetically encoded ion sensors to follow real time activation [35], and knockout constructs to examine loss of function phenotypes. More simply, gene knockout and mutagenesis could be used to validate neurological target sites of novel insecticidal compounds.

\section{Applications for malaria control}

Two general strategies are being pursued for control of malaria through the release of genetically modified (GM) mosquitoes. One involves reducing the effective population size of Anopheles mosquitoes (suppression), while the other aims at reducing the number of infectious bites people receive without affecting population size (modification).

\section{Population suppression strategies}

Generally, suppression strategies aim at reducing the vector population size by impairing their ability to successfully reproduce (reviewed in [36-38]). A combination of transposon and site directed genetic approaches contributed to create anopheline lines displaying population suppressive traits.

PiggyBac transformed lines were used to express homing nucleases that cleaved zygotic $\mathrm{X}$ chromosomes resulting in male sterility [39], which was further refined to bias production of male-only progeny [40]. Sex distortion was demonstrated again using transposon-mediated insertion of CRISPR/Cas9 components resulting in selective destruction of $X$ chromosome-bearing sperms [41]. Flight, and thus mating capability, has also been targeted through tTA regulated expression of apoptotic effectors in female flight muscles [23]. More recently, female-targeted suppression was reported in phiC31 cassette exchange-derived lines carrying CRISPR/Cas9 alleles that knocked out three ovarian genes in An. gambiae [20] rendering females essentially sterile. In the near future, the increasing knowledge of Anopheles sex determination $[9,42]$ and the improved capability of modifying the $Y$ chromosome [43] will greatly contribute to genetic sex distorting technologies.

\section{Population modification strategies}

While suppression strategies are based on introducing a significant fitness load into the population, modification (or replacement) approaches rely on the introgression of traits that impair parasite development in the vector to limit malaria transmission.

Various strategies for the creation of GM anophelines that are refractory to infection have been exploited and are reviewed in [44]. Successful parasite blocking relies on tightly regulated promoters 
driving precise temporal and spatial expression of antimalarial molecules in relevant tissues (usually midgut, haemolymph, and salivary glands) to coincide with parasite development following ingestion. To date, most strategies to modify transmission have relied on the use of transposon or integrasemediated strategies to express different effector genes including: endogenous or exogenous antimicrobial molecules; interaction inhibitor molecules; or the sustained activation of antiparasitic signalling pathways [16,44]. RNAi and then later CRISPR/Cas9-based NHEJ approaches have also been exploited to silence the parasite agonist fibrinogen-related protein-1 in An. gambiae [33,45]. While these strategies result in a significant decrease of Plasmodium prevalence and/or intensity of infection, they most often impose fitness costs that may also be indirectly responsible for parasite load reductions. A more specific anti-Plasmodium approach involves supplementing mosquito innate immunity by expressing single chain antibodies (scFvs) that target antigens expressed on the parasite surface during development within the mosquito [46]. In view of their specific blocking capacity and the fact that these mosquitoes appear as fit as wild type under the conditions tested [47], expression of multiplexed scFvs is perhaps the most promising current approach for population modification.

\section{Gene drives}

While proof-of-principle experiments for population suppression and modification have been successful, strategies that would rely on reiterative mass release of mosquitoes to spread a trait are very costly, and a drive system for the rapid introgression of favourable traits into a wild population from relatively low threshold numbers is likely to be more readily achieved and sustained.

Gene drives are based on genetic elements that are inherited with a frequency greater than Mendelian segregation rates, which allows them to spread in a population in relatively few generations, theoretically even against a degree of fitness cost [37] (Fig 3). While gene drives are not a new concept [48], the advent of programmable site-specific nucleases made their application more tractable $[37,49]$ The first proof of principle for a gene drive in anophelines was based on the expression of the homing nuclease I-Scel [27] targeting a modified transgenic GFP. Cage trials indicated high drive efficiency and opened the potential for similar systems to force desirable traits into populations. More recently, CRISPR/Cas9 was used to generate two further gene drives [50]. A population modification system was successfully developed in An. stephensi to drive scFvs directed against Plasmodium proteins into a caged population with $>99 \%$ efficiency [32]. Shortly after, a population suppression gene drive was described in An. gambiae that targeted one of the aforementioned candidate ovary specific genes for knockout and showed around $75 \%$ drive efficiency in caged populations [20].

These initial studies showcase the immense potential for transforming populations, but a number of significant hurdles need to be overcome before they can be used in real world scenarios for disease control. These issues and potential proposed solutions are extensively discussed in the indicated articles and include: technical obstacles, such as the generation of resistance to the drive [51,52]; concerns about standards for laboratory confinement [53,54]; the feasibility of conducting safe and ethical field trials [55] and options for recalling, limiting spread or stopping the drive once released [56]. In this context, modelling of long term population dynamics will be critical to predict potential epidemiological outcomes, as well as longer term effects on biodiversity and ecosystems [57-59].

While still in their infancy, CRISPR/Cas9-based gene drives hold out a promise of very powerful, costeffective and self-sustainable tools to be added to the malaria control toolbox. Moreover, the ongoing advances in genetic technologies enable the discovery of gene functions that will inform and direct improvements in current intervention strategies through greater knowledge of vector biology.

\section{Acknowledgements}


We very much thank Marta Miguel Andres (currently at Joerg Albert's lab in Ear Institute, UCL, UK) for providing the unpublished Figure 2D. We are also indebted to Prof David Sattelle and Dr Grazia Camarda for providing very useful comments on the manuscript. 
Table 1. Comparison of genetic manipulation techniques in Anopheles

\begin{tabular}{|c|c|c|c|c|c|c|}
\hline \multirow{2}{*}{$\begin{array}{c}\text { Basis of } \\
\text { modification }\end{array}$} & \multicolumn{2}{|c|}{ RNA Interference } & \multirow[t]{2}{*}{ Transposon } & \multirow[t]{2}{*}{ Integrase/recombinase } & \multirow{2}{*}{$\begin{array}{c}\text { Bipartite expression } \\
\text { systems }\end{array}$} & \multirow[t]{2}{*}{ Site specific nucleases } \\
\hline & Transient & Stable & & & & \\
\hline System used & $\begin{array}{l}\text { Synthetic dsRNA } \\
\text { injection/feeding }\end{array}$ & $\begin{array}{l}\text { Transgenic } \\
\text { expression of } \\
\text { hairpin RNA }\end{array}$ & $\begin{array}{c}\text { Minos } \\
\text { PiggyBac }\end{array}$ & phiC31 & $\begin{array}{c}\mathrm{tTA} \\
\text { Gal4/UAS } \\
\mathrm{Q}\end{array}$ & $\begin{array}{l}\text { HEGS } \\
\text { TALENS } \\
\text { CRISPR }\end{array}$ \\
\hline $\begin{array}{l}\text { Anopheles } \\
\text { Species } \\
\text { modified }\end{array}$ & $\begin{array}{l}\text { An. gambiae/coluzzii } \\
\text { [3] } \\
\text { An. stephensi [60] } \\
\text { An. arabiensis [9] } \\
\text { An. dirus [61] } \\
\text { An. sinensis [10] } \\
\text { An. aquasalis [62] }\end{array}$ & An. stephensi [63] & $\begin{array}{l}\text { An. gambiae/coluzzii [13] } \\
\text { An. stephensi [14] } \\
\text { An. albimanus [64] }\end{array}$ & $\begin{array}{l}\text { An. gambiae/coluzzii [19] } \\
\text { An. stephensi [47] }\end{array}$ & $\begin{array}{l}\text { An. gambiae/coluzzii } \\
{[24,25]} \\
\text { An. stephensi [22] }\end{array}$ & $\begin{array}{l}\text { An. } \\
\text { gambiae/coluzzi[20,27,29,31] } \\
\text { An. stephensi [32] } \\
\text { An. funestus [31,32] } \\
\text { An. albimanus [31,32] }\end{array}$ \\
\hline $\begin{array}{c}\text { Site of } \\
\text { integration }\end{array}$ & $\mathrm{N} / \mathrm{A}$ & $\begin{array}{l}\text { Dependant on } \\
\text { integration system } \\
\text { used. }\end{array}$ & $\begin{array}{l}\text { Essentially random } \\
\text { (TTAA for piggyBac, TA } \\
\text { for Minos) }\end{array}$ & $\begin{array}{l}\text { Site directed into pre- } \\
\text { existing attP tagged sites } \\
\text { in the genome. }\end{array}$ & $\begin{array}{l}\text { Dependant on } \\
\text { integration system } \\
\text { used. }\end{array}$ & $\begin{array}{l}\text { Predetermined, targeted } \\
\text { genomic sites. }\end{array}$ \\
\hline Advantages & $\begin{array}{l}\text { Rapid; } \\
\text { Relatively high } \\
\text { throughput; } \\
\text { Can be targeted to } \\
\text { adult stages. }\end{array}$ & $\begin{array}{l}\text { Tissue and temporal } \\
\text { specific knockdown; } \\
\text { Heritable } \\
\text { knockdown. }\end{array}$ & $\begin{array}{l}\text { Unbiased integration; } \\
\text { Suitable for locus, fitness, } \\
\text { and enhancer/exon } \\
\text { screening; } \\
\text { Can be remobilised for } \\
\text { mutation screens. }\end{array}$ & $\begin{array}{l}\text { Comparative analysis of } \\
\text { genes expressed at same } \\
\text { genomic locus. } \\
\text { Greater cargo size than } \\
\text { TEs. }\end{array}$ & $\begin{array}{l}\text { Suitable for toxic and } \\
\text { developmental genes; } \\
\text { Flexible analysis of } \\
\text { tissue specific } \\
\text { phenotypes. }\end{array}$ & $\begin{array}{l}\text { Vast array of mutations and } \\
\text { expression regulated lines } \\
\text { can be created. Can utilise } \\
\text { endogenously located } \\
\text { promoters and genes for } \\
\text { mutagenesis. } \\
\text { Potential drives are easily } \\
\text { designed (CRISPR/Cas9). }\end{array}$ \\
\hline Drawbacks & $\begin{array}{l}\text { Transient; } \\
\text { One generation only; } \\
\text { Does not target all } \\
\text { genes/tissues equally. }\end{array}$ & $\begin{array}{l}\text { Longer time to } \\
\text { generate through } \\
\text { transgenesis; } \\
\text { May be selected } \\
\text { against during } \\
\text { maintenance. }\end{array}$ & $\begin{array}{l}\text { Positional variegation of } \\
\text { expression; } \\
\text { Limited number of } \\
\text { promoters available; } \\
\text { Limited cargo size } 10-12 \\
\text { Kb. }\end{array}$ & $\begin{array}{l}\text { Need to create docking } \\
\text { lines initially; } \\
\text { Limited number of } \\
\text { promoters available. }\end{array}$ & $\begin{array}{l}\text { Multiple lines need to } \\
\text { be maintained. } \\
\text { Limited number of } \\
\text { promoters available. }\end{array}$ & $\begin{array}{l}\text { Except CRISPR/Cas9, quite } \\
\text { extensive engineering } \\
\text { required. } \\
\text { Normally creates null } \\
\text { mutations, potentially } \\
\text { inviable if dominant. }\end{array}$ \\
\hline
\end{tabular}


Figure 1.

\section{A) RNA interference (RNAi)}

Synthetic double stranded RNA (dsRNA) homologous to the target gene transcript are typically injected into the mosquito haemocoel, where they are taken up by some tissue types (including fat body, oenocytes, haemocytes and midgut) and cleaved by the Dicer ribonuclease into small ( $20 \mathrm{nt})$ interfering dsRNAs (siRNAs). These are incorporated into the RNA-induced silencing complex (RISC) which degrades one strand, while the other strand directs the silencing complex to the target mRNA. After strict complementarity-based binding, the RISC nuclease Argonaute (Argo) catalyzes the cleavage and thus inactivation of the mRNA. Several days are left to allow for protein turnover, after which phenotypic assays are performed versus control mosquitoes.

\section{B) The PiggyBac Transposon}

Embryos are injected at the posterior pole (germline localization) with a donor plasmid carrying the transgene flanked by piggyBac inverted terminal repeats (ITR) and a transient source of transposase (TR), either plasmid encoded, mRNA or purified protein. The TR recognizes the ITRs and catalyzes the "cut and paste" of the cassette into a "random" TTAA sequence in the germline chromosome. Insertion preserves the ITRs and if transposase is supplied in later generations, remobilization of the cassette can occur. Modification of the germline is passed onto the next generation and can be followed by monitoring expression of a fluorescent marker gene (such as dsRed) linked to the transgene. Transgenes will normally consist of a promoter to drive tissue specific expression of a downstream effector gene followed by a signal sequence to terminate transcription.

\section{C) The PhiC31/att system.}

Embryos of phiC31 docking lines carrying (i) one or (ii) two attP sites in a defined chromosomal locus are injected with a donor plasmid carrying a transgene and fluorescent protein gene flanked by one (i) or two (ii) attB sites and a transient source of integrase (INT). i) Integration. The integrase catalyzes the recombination at the att sites resulting in the integration of the entire donor plasmid DNA into the genomic docking site. ii) Cassette exchange. The integrase catalyzes double crossover recombination the att sites resulting in the swap of the originally integrated cassette and removal of the plasmid backbone. The alternative transgenes are generally marked with distinct fluorescent proteins to facilitate inheritance screening. Recombination results in the formation of hybrid sites attR and attL that are resistant to remobilization.

\section{D) Binary expression systems.}

In anophelines, three types of bipartite expression systems have been developed: the tTA/tetO, GAL4/UAS, and Q systems. All comprise of separate driver lines that express a transcriptional activator factor under regulation of a desired promoter, and responder lines carrying the gene of interest downstream of corresponding activation factor binding sites. When the two components are brought together in the progeny of driver and responder crosses the transgene will be expressed and potential phenotype observed. The driver construct dictates the temporal and spatial pattern of expression; the responder construct determines which protein is expressed. The lines are usually marked with different fluorophores to facilitate screening for co-inheritance. GAL4, QF and tTA are transcriptional activators from yeast, Neurospora crassa, and E. coli respectively. UAS, QUAS and tetO are their corresponding binding sites. The tTA systems are responsive to tetracycline, and so gene expression can be switched on/off by supplying mosquitoes with tetracycline analogues. The GAL4 and QF system can be switched off by co-expressing either Gal80 or Q-S suppressors respectively in the same tissues (although this has not yet been performed in anophelines).

E) CRISPR (Clustered Regularly Interspaced Short Palindromic Repeats)/Cas9 site-specific mutagenesis. During embryo injection, a transient source of site-specific endonuclease Cas9 is provided alongside a short (-20 nt) guide RNA (gRNA), either in vitro transcribed, plasmid encoded or synthesised, which has perfect complementarity to the sequence targeted for modification. The target site is defined by and lies immediately adjacent to a protospacer adjacent motif (PAM) with sequence NGG. The Cas9 is directed to a desired genomic target by the gRNA and creates a double stranded DNA break that is precisely located $3 \mathrm{bp}$ upstream of the PAM site. Chromosome integrity is restored by the cell DNA repairing machinery using either of two different mechanisms: Non-Homologous End Joining (NHEJ) or Homology Directed Repair (HDR). NHEJ results from the imprecise ligation of the broken DNA extremities which in most cases causes frameshift indel mutations and thus gene knockout when occurring in coding regions. HDR occurs when donor DNA having significant homology surrounding the breakpoint is used by the cell as a template to repair the break. By including a desired cargo (e.g. promoters, markers, mutagenised genes, tags) flanked by sequences homologous to the surrounding breakpoint locus into the co-injected donor DNA template, these are precisely incorporated into the genomic site via homologous recombination. 
Figure 2. Anopheles chordotonal organs

A, B, C: Piggybac transformed An. gambiae carrying an alpha-tubulin promoter fusion with eGFP and dsRed fluorescent marker driven by the synthetic neuronal promoter Pax3. Fluorescently tagged chordotonal (mechanosensory) organs (yellow arrows) are visible through the cuticle and hence readily available for real time vital imaging in A) larval abdomen and thorax, B) dorsal side of the adult thorax and C) the junction of thorax and femur [65]. D) Immuno-stained section of Johnston's organ (JO) or the male mosquito antennal 'ear'; phalloidin (actin blue) and anti-HRP (neuronal membrane marker - red), courtesy of Marta Andres Miguel [66]. The JO is a complex chordotonal organ located at the base of the flagellum (distal part of antennae) and contains over 15,000 auditory neurons that transduce sound-induced mechanical vibrations from the flagellum into electrical signals.

The auditory neurons are bipolar and monodendritic, with ciliated dendritic outer segments pointing towards and indirectly coupled to the base of the flagellum. The auditory neurons are arranged in groups of 2-3 and form together with two accessory cell functional units called scolopidia. Mosquitoes mate in swarms, and males detect potential mates by tuning into the wing-beat frequency of conspecific females. Analysis of mosquito hearing has many avenues for mosquito control, including insecticides that target signal transduction, genetic control targeting mating success and acoustic traps.

Figure 3. CRISPR/Cas9-based gene drives.

To create a gene drive, the germline transformation is designed so that the gRNA and Cas9 are included in the knockin construct and so are incorporated into the cleaved target site on the chromosome or so called 'drive' allele by HDR. The chromosomally encoded Cas9 will then cut the wild type allele on the homologous chromosome, which by HDR is converted into another drive allele. By using promoter sequences to drive nuclease expression in early germline tissues, most or all of the gametes will thus contain a drive allele which will be inherited by up to $100 \%$ of the subsequent progeny. Other genes (cargo) can be included along with the nuclease to encode desired traits that will be also inherited at the same frequency as the drive, and/or if the target sequence is within a coding region then this gene will be knocked out simultaneously during HDR.

In the suppression drives designed to date, the inheritance of the drive allele disrupts the coding region of genes that regulate reproductive capacity. If Cas9 activity is restricted to the germline, then fertile somatic heterozygotes, will carry homozygous gene drives in the germline and pass on the drive at super-Mendelian rates. In time, the increase of drive allele frequency will produce a predominance of somatic infertile homozygotes that results in a crash of population size.

In modification drives, genes conferring refractoriness to malaria are inherited at super-Mendelian rates along with the drive. Depending on the degree of fitness costs due to modification, the antimalarial trait will introgress into the population while the overall size will not be affected. 


\section{References}

1. Organization WH: World Malaria Report 2017. WHO Global Malaria Programme. Edited by: WHO Geneva; 2018.

2. The Anopheles gambiae Genomes C: Genetic diversity of the African malaria vector Anopheles gambiae. Nature 2017, 552:96.

3. Catteruccia F, Levashina EA: RNAi in the malaria vector, Anopheles gambiae. Methods $\mathrm{Mol}$ Biol 2009, 555:63-75.

4 •. Simoes ML, Mlambo G, Tripathi A, Dong Y, Dimopoulos G: Immune Regulation of Plasmodium Is Anopheles Species Specific and Infection Intensity Dependent. MBio 2017, 8.

This study describes the extensive use of RNAi to examine host factors influencing vectorial capacity. Immune responses of New and Old World Anopheles were compared after infection with human and rodent malaria parasites. The work indicated differential agonistic and antagonistic effector gene regulation of parasite refractoriness in the two species, with infection intensity being a major parameter in parasite melanisation rather than mosquito/parasite species combinations.

5. Osta MA, Christophides GK, Vlachou D, Kafatos FC: Innate immunity in the malaria vector Anopheles gambiae: comparative and functional genomics. J Exp Biol 2004, 207:25512563.

6. Blandin S, Shiao SH, Moita LF, Janse CJ, Waters AP, Kafatos FC, Levashina EA: Complementlike protein TEP1 is a determinant of vectorial capacity in the malaria vector Anopheles gambiae. Cell 2004, 116:661-670.

7. Ingham VA, Pignatelli P, Moore JD, Wagstaff S, Ranson H: The transcription factor Maf-S regulates metabolic resistance to insecticides in the malaria vector Anopheles gambiae. BMC Genomics 2017, 18:669.

8. Doran CR, Estevez-Lao TY, Hillyer JF: Mosquito aging modulates the heart rate and the proportional directionality of heart contractions. J Insect Physiol 2017, 101:47-56.

$9 \bullet \bullet$. Krzywinska E, Dennison NJ, Lycett GJ, Krzywinski J: A maleness gene in the malaria mosquito Anopheles gambiae. Science 2016, 353:67-69.

The paper describes the discovery of the primary switch (Yob) of sex determination in An. gambiae through differential RNA-seq analysis after individual sexing of embryos. Analysis of gene function through RNAi and mRNA microinjection in embryos supported a role for yob in dosage compensation of $X$ linked expression through male specific lethality of knockdown and female lethality of ectopic expression, and opened an avenue to dissect this unexplored pathway in Anopheles. The discovery of a transcriptionally active region of the Y Chromosome also created further target sites for sex ratio distorting gene drives.

10. Du MH, Yan ZW, Hao YJ, Yan ZT, Si FL, Chen B, Qiao L: Suppression of Laccase 2 severely impairs cuticle tanning and pathogen resistance during the pupal metamorphosis of Anopheles sinensis (Diptera: Culicidae). Parasit Vectors 2017, 10:171.

11. Mysore K, Hapairai LK, Sun L, Harper EI, Chen Y, Eggleson KK, Realey JS, Scheel ND, Severson DW, Wei N, et al.: Yeast interfering RNA larvicides targeting neural genes induce high rates of Anopheles larval mortality. Malar J 2017, 16:461. 
12. Lycett GJ, McLaughlin LA, Ranson H, Hemingway J, Kafatos FC, Loukeris TG, Paine MJ: Anopheles gambiae $\mathrm{P} 450$ reductase is highly expressed in oenocytes and in vivo knockdown increases permethrin susceptibility. Insect Mol Biol 2006, 15:321-327.

13. Grossman GL, Rafferty CS, Clayton JR, Stevens TK, Mukabayire O, Benedict MQ: Germline transformation of the malaria vector, Anopheles gambiae, with the piggyBac transposable element. Insect Mol Biol 2001, 10:597-604.

14. Catteruccia F, Nolan T, Loukeris TG, Blass C, Savakis C, Kafatos FC, Crisanti A: Stable germline transformation of the malaria mosquito Anopheles stephensi. Nature 2000, 405:959-962.

$15 \bullet$. Adelman ZN, Basu S, Myles KM: Chapter 8 - Gene Insertion and Deletion in Mosquitoes. In Genetic Control of Malaria and Dengue. Edited by: Academic Press; 2016:139-168.

Extensive review of the tools available for genome modification of mosquitoes that incudes species beyond the Anopheles genus, published prior to the first uses of CRISPR/Cas9 for gene drives. Includes sections that explain the available promoters to drive expression, mechanisms of transformation and the advantages and disadvantages of alternative approaches.

16. Wang S, Jacobs-Lorena M: Genetic approaches to interfere with malaria transmission by vector mosquitoes. Trends Biotechnol 2013, 31:185-193.

17. O'Brochta DA, Pilitt KL, Harrell RA, 2nd, Aluvihare C, Alford RT: Gal4-based enhancertrapping in the malaria mosquito Anopheles stephensi. G3 (Bethesda) 2012, 2:13051315.

$18 \bullet$ •. Adolfi A, Pondeville E, Lynd A, Bourgouin C, Lycett GJ: Multi-tissue GAL4-mediated gene expression in all Anopheles gambiae life stages using an endogenous polyubiquitin promoter. Insect Biochem Mol Biol 2018, 96:1-9.

The study describes the use of transposon based analysis to identify an endogenous ubiquitous promoter in An. gambiae and to define genomic sites that are permissive to the expression of Gal4 drivers in multiple tissues.

19. Meredith JM, Underhill A, McArthur CC, Eggleston P: Next-generation site-directed transgenesis in the malaria vector mosquito Anopheles gambiae: self-docking strains expressing germline-specific phiC31 integrase. PLoS One 2013, 8:e59264.

20 ••. Hammond A, Galizi R, Kyrou K, Simoni A, Siniscalchi C, Katsanos D, Gribble M, Baker D, Marois E, Russell S, et al.: A CRISPR-Cas9 gene drive system targeting female reproduction in the malaria mosquito vector Anopheles gambiae. Nat Biotechnol 2016, 34:78-83.

The first report of the use of CRISPR/Cas9 in An. gambiae, as well as its development as a population suppressive gene drive system. A mixture of TALEN and Cas9 based approaches were used to examine sterility phenotypes (whether haplo-sufficient) of knockout of three ovary specific genes and concomitant knockin of a GFP marker and phiC31 docking sites. Moreover, RMCE was then used to insert Cas9/gRNA alleles at the same loci to assess the efficiency of suppressive gene drives targeting these genes. One of the loci was modelled and proven in cage testing to show an increase in allele frequency over 4 generations. Brief mention is made of the problem of alleles to drive. 
21. Volohonsky G, Terenzi O, Soichot J, Naujoks DA, Nolan T, Windbichler N, Kapps D, Smidler AL, Vittu A, Costa G, et al.: Tools for Anopheles gambiae Transgenesis. G3 (Bethesda) 2015, 5:1151-1163.

22. Lycett GJ, Kafatos FC, Loukeris TG: Conditional expression in the malaria mosquito Anopheles stephensi with Tet-On and Tet-Off systems. Genetics 2004, 167:1781-1790.

23. Marinotti O, Jasinskiene N, Fazekas A, Scaife S, Fu G, Mattingly ST, Chow K, Brown DM, Alphey L, James AA: Development of a population suppression strain of the human malaria vector mosquito, Anopheles stephensi. Malar J 2013, 12:142.

24. Lynd A, Lycett GJ: Development of the bi-partite Gal4-UAS system in the African malaria mosquito, Anopheles gambiae. PLoS One 2012, 7:e31552.

25 •. Riabinina O, Task D, Marr E, Lin CC, Alford R, O'Brochta DA, Potter CJ: Organization of olfactory centres in the malaria mosquito Anopheles gambiae. Nat Commun 2016, 7:13010.

The first paper describing the use of the $Q$ bipartite system in Anopheles. PiggyBac insertions created 4 different sets of neuronal promoter QF driver lines and a responder line carrying QUAS-mCD8 membrane GFP. One set of the driver lines was confirmed to recapitulate the expression pattern expected, and was elegantly used to fluorescently tag the neurons expressing the odorant receptor co-receptor, orco. The lack of activity in the 3 other drivers illustrates the difficulty in defining functional promoter sequences in mosquitoes.

26 •. Guha TK, Wai A, Hausner G: Programmable Genome Editing Tools and their Regulation for Efficient Genome Engineering. Comput Struct Biotechnol J 2017, 15:146-160.

Review paper that gives greater detail on the mechanics of the gene editing tools, as well as forecasting the potential modifications of these systems that will provide switches to alter the temporal control. These tools may have future applications in mosquitoes.

$27 \bullet$. Windbichler N, Menichelli M, Papathanos PA, Thyme SB, Li H, Ulge UY, Hovde BT, Baker D, Monnat RJ, Jr., Burt A, et al.: A synthetic homing endonuclease-based gene drive system in the human malaria mosquito. Nature 2011, 473:212-215.

First report of a gene drive system in Anopheles. This was based on the use of the male germline expressed homing endonuclease targeting a GFP transgene, and demonstrated the proof of principle for drive systems to invade cage populations

28. Yamamoto DS, Sumitani M, Hatakeyama M, Matsuoka H: Malaria infectivity of xanthurenic acid-deficient anopheline mosquitoes produced by TALEN-mediated targeted mutagenesis. Transgenic Res 2018, 27:51-60.

29 •. Volohonsky G, Hopp AK, Saenger M, Soichot J, Scholze H, Boch J, Blandin SA, Marois E: Transgenic Expression of the Anti-parasitic Factor TEP1 in the Malaria Mosquito Anopheles gambiae. PLoS Pathog 2017, 13:e1006113.

This paper uses multiple genetic tools to address the functional activity of immune factor TEP1 in plasmodium infection. Most novel is the first use of a deactivated site specific nuclease (TALEN) to modulate expression of the native gene. Although only moderate enhancement of expression was achieved that didn't impact plasmodium development, this is a significant step on the way to regulate endogenous expression in anophelines.

30. Smidler AL, Terenzi O, Soichot J, Levashina EA, Marois E: Targeted mutagenesis in the malaria mosquito using TALE nucleases. PLoS One 2013, 8:e74511. 
$31 \bullet \bullet$. Li M, Akbari OS, White BJ: Highly Efficient Site-Specific Mutagenesis in Malaria Mosquitoes Using CRISPR. G3 (Bethesda) 2018, 8:653-658.

Application of CRISPR/Cas9 NHEJ knockouts to multiple Anopheles species targeting the $X$ linked white gene, that allowed easily scorable phenotype and differentiation between biallelic and single allele mutagenesis. Provides standard protocol to inject in vitro synthesised gRNAs and commercial purified Cas9 protein, and suggests a very high frequency of mutation.

$32 \bullet \bullet$. Gantz VM, Jasinskiene N, Tatarenkova O, Fazekas A, Macias VM, Bier E, James AA: Highly efficient Cas9-mediated gene drive for population modification of the malaria vector mosquito Anopheles stephensi. Proc Natl Acad Sci U S A 2015, 112:E6736-6743.

First report of CRISPR/Cas9 HDR-based germline transformation and CRISPR/Cas9-based gene drive in Anopheles. The paper describes efficient gene drive of antimalarial single chain antibodies through the male line, with near Mendelian inheritance through female lines, suggesting that strict germline expression is required for effective drives.

$33 \bullet$ • Dong Y, Simoes ML, Marois E, Dimopoulos G: CRISPR/Cas9 -mediated gene knockout of Anopheles gambiae FREP1 suppresses malaria parasite infection. PLoS Pathog 2018, 14:e1006898.

Following on from RNAi approaches published previously, this uses work CRISPR/Cas9 knockout to demonstrate the role of FREP1 as a positive regulator of Plasmodium infection in the midgut of An. gambiae. Fitness costs of the null mutation may contribute to changes of parasite burden seen in the knockouts.

34. Wang $\mathrm{Y}$, Wang F, Wang R, Zhao $\mathrm{P}$, Xia Q: 2A self-cleaving peptide-based multi-gene expression system in the silkworm Bombyx mori. Sci Rep 2015, 5:16273.

35. Bui M, Shyong J, Lutz EK, Yang T, Li M, Truong K, Arvidson R, Buchman A, Riffell JA, Akbari OS: Live calcium imaging of Aedes aegypti neuronal tissues reveals differential importance of chemosensory systems for life-history-specific foraging strategies. bioRxiv 2018.

36. Hammond AM, Galizi R: Gene drives to fight malaria: current state and future directions. Pathog Glob Health 2017, 111:412-423.

37. Godfray HCJ, North A, Burt A: How driving endonuclease genes can be used to combat pests and disease vectors. BMC Biol 2017, 15:81.

$38 \bullet \bullet$. Burt A, Coulibaly M, Crisanti A, Diabate A, Kayondo JK: Gene drive to reduce malaria transmission in sub-Saharan Africa. Journal of Responsible Innovation 2018, 5:S66-S80.

Discusses the different targets for gene drive that have been proposed for malaria control in Africa, the attention needed to monitor for resistance development and the considerations required by all concerned for their future field deployment.

39. Windbichler N, Papathanos PA, Crisanti A: Targeting the $\mathbf{X}$ chromosome during spermatogenesis induces $\mathrm{Y}$ chromosome transmission ratio distortion and early dominant embryo lethality in Anopheles gambiae. PLoS Genet 2008, 4:e1000291.

40. Galizi R, Doyle LA, Menichelli M, Bernardini F, Deredec A, Burt A, Stoddard BL, Windbichler $\mathrm{N}$, Crisanti A: A synthetic sex ratio distortion system for the control of the human malaria mosquito. Nat Commun 2014, 5:3977.

41 •. Galizi R, Hammond A, Kyrou K, Taxiarchi C, Bernardini F, O'Loughlin SM, Papathanos PA, Nolan T, Windbichler N, Crisanti A: A CRISPR-Cas9 sex-ratio distortion system for genetic control. Sci Rep 2016, 6:31139. 
Describes the production of a CRISPR/Cas9-based version of the X-shredder strategy introduced in [40]. This population suppression approach is based on the selective disruption of the $X$ chromosome during male spermatogenesis to create strongly biased male-only progeny. The strategy may also be directly adaptable to other species with the same sex determination.

42. Criscione F, Qi Y, Tu Z: GUY1 confers complete female lethality and is a strong candidate for a male-determining factor in Anopheles stephensi. Elife 2016, 5.

43. Bernardini F, Galizi R, Menichelli M, Papathanos PA, Dritsou V, Marois E, Crisanti A, Windbichler N: Site-specific genetic engineering of the Anopheles gambiae $Y$ chromosome. Proc Natl Acad Sci U S A 2014, 111:7600-7605.

44 •. Carballar-Lejarazu R, James AA: Population modification of Anopheline species to control malaria transmission. Pathog Glob Health 2017, 111:424-435.

Describes the key challenges of taking population modification gene drive strategies into the field. There is a strong emphasis on appropriate target product profiles necessary to generate suitable field ready strains for release, that take into consideration safety and efficacy, as well as the social science and ethical analysis needed to meet regulatory and stake holder requirements.

45. Zhang G, Niu G, Franca CM, Dong Y, Wang X, Butler NS, Dimopoulos G, Li J: Anopheles Midgut FREP1 Mediates Plasmodium Invasion. J Biol Chem 2015, 290:16490-16501.

46. Isaacs AT, Li F, Jasinskiene N, Chen X, Nirmala X, Marinotti O, Vinetz JM, James AA: Engineered resistance to Plasmodium falciparum development in transgenic Anopheles stephensi. PLoS Pathog 2011, 7:e1002017.

47. Isaacs AT, Jasinskiene N, Tretiakov M, Thiery I, Zettor A, Bourgouin C, James AA: Transgenic Anopheles stephensi coexpressing single-chain antibodies resist Plasmodium falciparum development. Proc Natl Acad Sci U S A 2012, 109:E1922-1930.

48. Ribeiro JM, Kidwell MG: Transposable elements as population drive mechanisms: specification of critical parameter values. J Med Entomol 1994, 31:10-16.

49. Macias VM, Ohm JR, Rasgon JL: Gene Drive for Mosquito Control: Where Did It Come from and Where Are We Headed? Int J Environ Res Public Health 2017, 14.

50. Gantz VM, Bier E: The dawn of active genetics. Bioessays 2016, 38:50-63.

51. Marshall JM, Buchman A, Sanchez CH, Akbari OS: Overcoming evolved resistance to population-suppressing homing-based gene drives. Sci Rep 2017, 7:3776.

52 •. Hammond AM, Kyrou K, Bruttini M, North A, Galizi R, Karlsson X, Kranjc N, Carpi FM, D'Aurizio R, Crisanti A, et al.: The creation and selection of mutations resistant to a gene drive over multiple generations in the malaria mosquito. PLoS Genet 2017 , 13:e1007039.

Following on from the demonstration of gene drive targeting female fertility, this paper provides the first extensive analysis of the formation of alleles that are resistant to a CRISPR/Cas9-based suppressive gene drive in An. gambiae, and suggests ways that they may be overcome. 53. Benedict MQ, Burt A, Capurro ML, De Barro P, Handler AM, Hayes KR, Marshall JM, Tabachnick WJ, Adelman ZN: Recommendations for Laboratory Containment and Management of Gene Drive Systems in Arthropods. Vector Borne Zoonotic Dis 2018, 18:2-13. 
54. Adelman ZN, Pledger D, Myles KM: Developing standard operating procedures for gene drive research in disease vector mosquitoes. Pathog Glob Health 2017, 111:436-447.

55. Kolopack PA, Lavery JV: Informed consent in field trials of gene-drive mosquitoes. Gates Open Res 2017, 1:14.

56. Min J, Smidler AL, Najjar D, Esvelt KM: Harnessing gene drive. Journal of Responsible Innovation 2018, 5:S40-S65.

57. Lambert B, North A, Burt A, Godfray HCJ: The use of driving endonuclease genes to suppress mosquito vectors of malaria in temporally variable environments. Malar J 2018, 17:154.

58 • . Eckhoff PA, Wenger EA, Godfray HC, Burt A: Impact of mosquito gene drive on malaria elimination in a computational model with explicit spatial and temporal dynamics. Proc Natl Acad Sci U S A 2017, 114:E255-E264.

Mathematical modelling of the long-term population dynamics of a variety of different suppression and modification gene drives in anopheline vectors. Provides insights into the effect of different drive parameters, including homing rate, seasonality, and release numbers on gene drive fate and likelihood of elimination in targeted areas.

59. Beaghton A, Hammond A, Nolan T, Crisanti A, Godfray HC, Burt A: Requirements for Driving Antipathogen Effector Genes into Populations of Disease Vectors by Homing. Genetics 2017, 205:1587-1596.

60. Pike A, Vadlamani A, Sandiford SL, Gacita A, Dimopoulos G: Characterization of the Rel2regulated transcriptome and proteome of Anopheles stephensi identifies new antiPlasmodium factors. Insect Biochem Mol Biol 2014, 52:82-93.

61. Wang Y, Wang Y, Zhang J, Xu W, Zhang J, Huang FS: Ability of TEP1 in intestinal flora to modulate natural resistance of Anopheles dirus. Exp Parasitol 2013, 134:460-465.

62. Bahia AC, Oliveira JH, Kubota MS, Araujo HR, Lima JB, Rios-Velasquez CM, Lacerda MV, Oliveira PL, Traub-Cseko YM, Pimenta PF: The role of reactive oxygen species in Anopheles aquasalis response to Plasmodium vivax infection. PLoS One 2013, 8:e57014.

63. Brown AE, Bugeon L, Crisanti A, Catteruccia F: Stable and heritable gene silencing in the malaria vector Anopheles stephensi. Nucleic Acids Res 2003, 31:e85.

64. Perera OP, Harrell IR, Handler AM: Germ-line transformation of the South American malaria vector, Anopheles albimanus, with a piggyBac/EGFP transposon vector is routine and highly efficient. Insect Mol Biol 2002, 11:291-297.

65. Lycett GJ, Amenya D, Lynd A: The Anopheles gambiae alpha-tubulin-1b promoter directs neuronal, testes and developing imaginal tissue specific expression and is a sensitive enhancer detector. Insect Mol Biol 2012, 21:79-88.

66. Andrés M, Seifert M, Spalthoff C, Warren B, Weiss L, Giraldo D, Winkler M, Pauls S, Göpfert Martin C: Auditory Efferent System Modulates Mosquito Hearing. Current Biology 2016, 26:2028-2036. 


\section{A) RNA Interference (RNAi)}

B) Piggybac Transposon

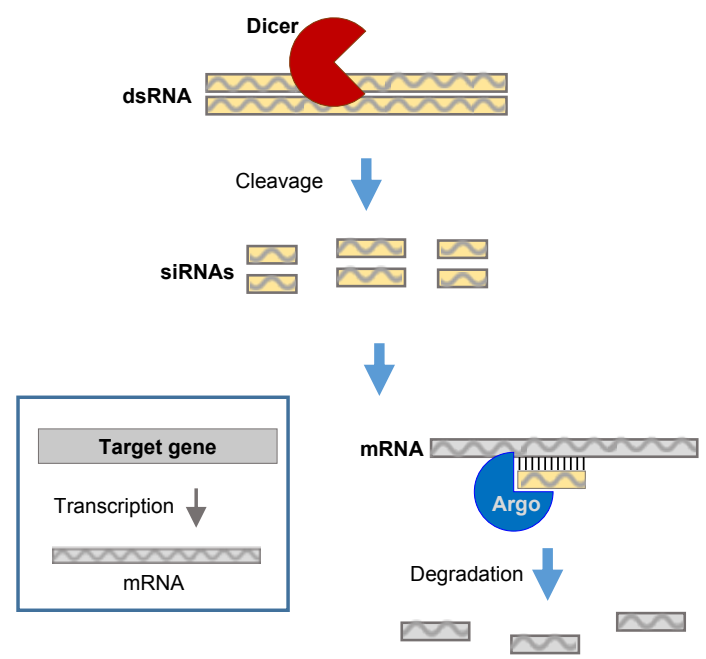

C) PhiC31 site directed recombination

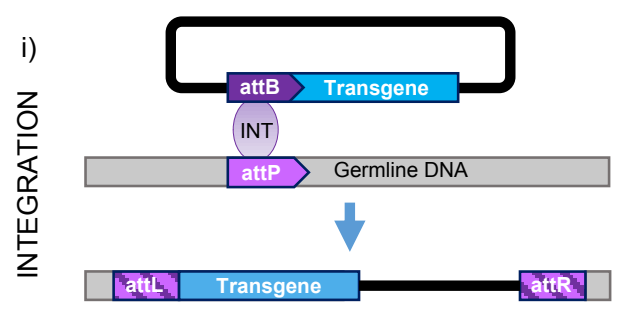

\section{D) Binary expression systems}
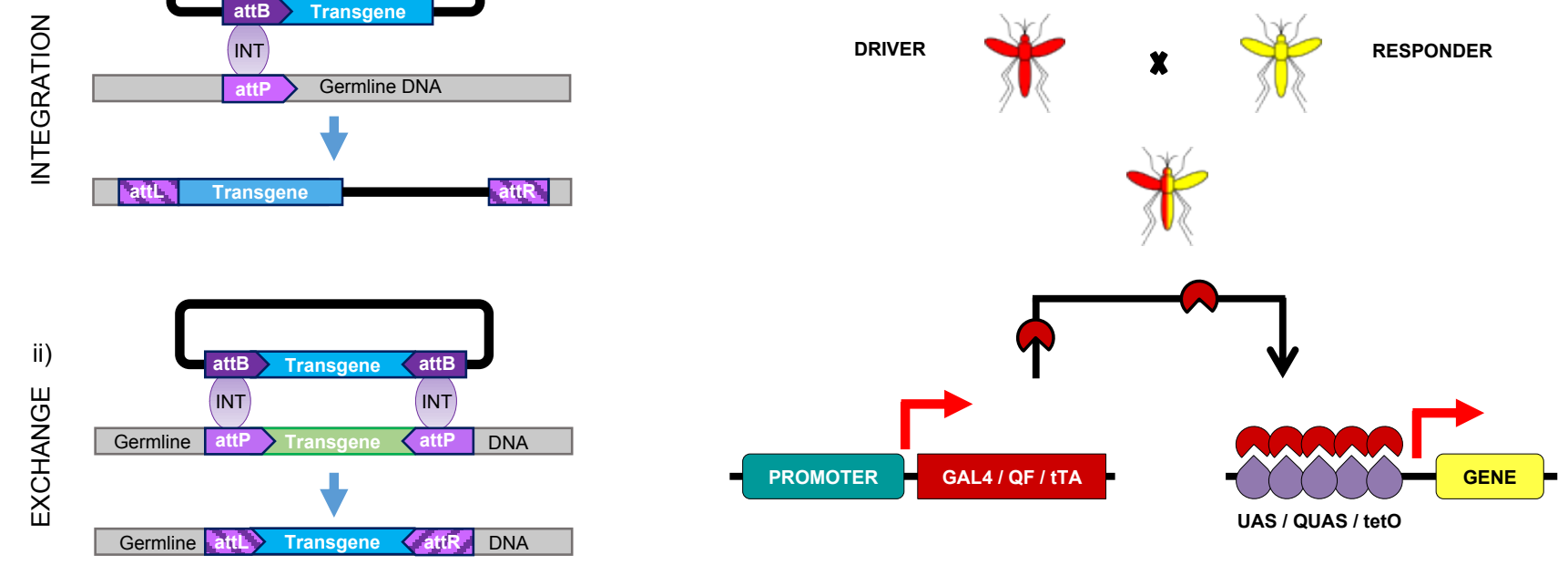

E) CRISPR/Cas9 site-specific mutagenesis

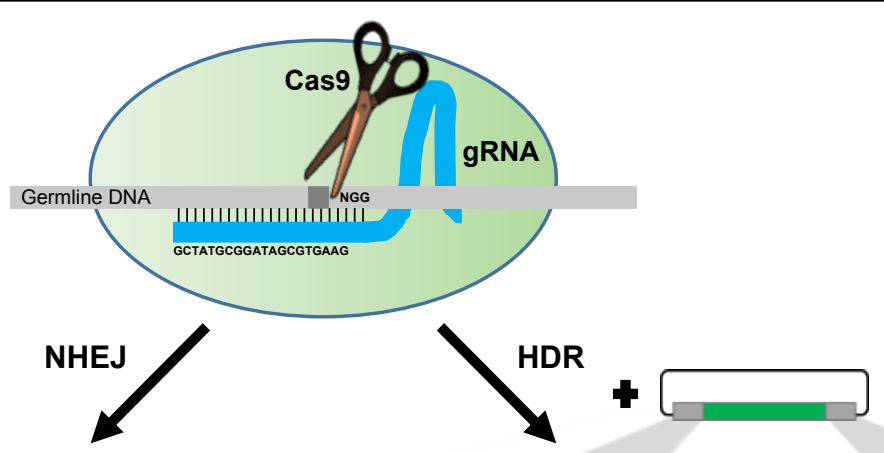




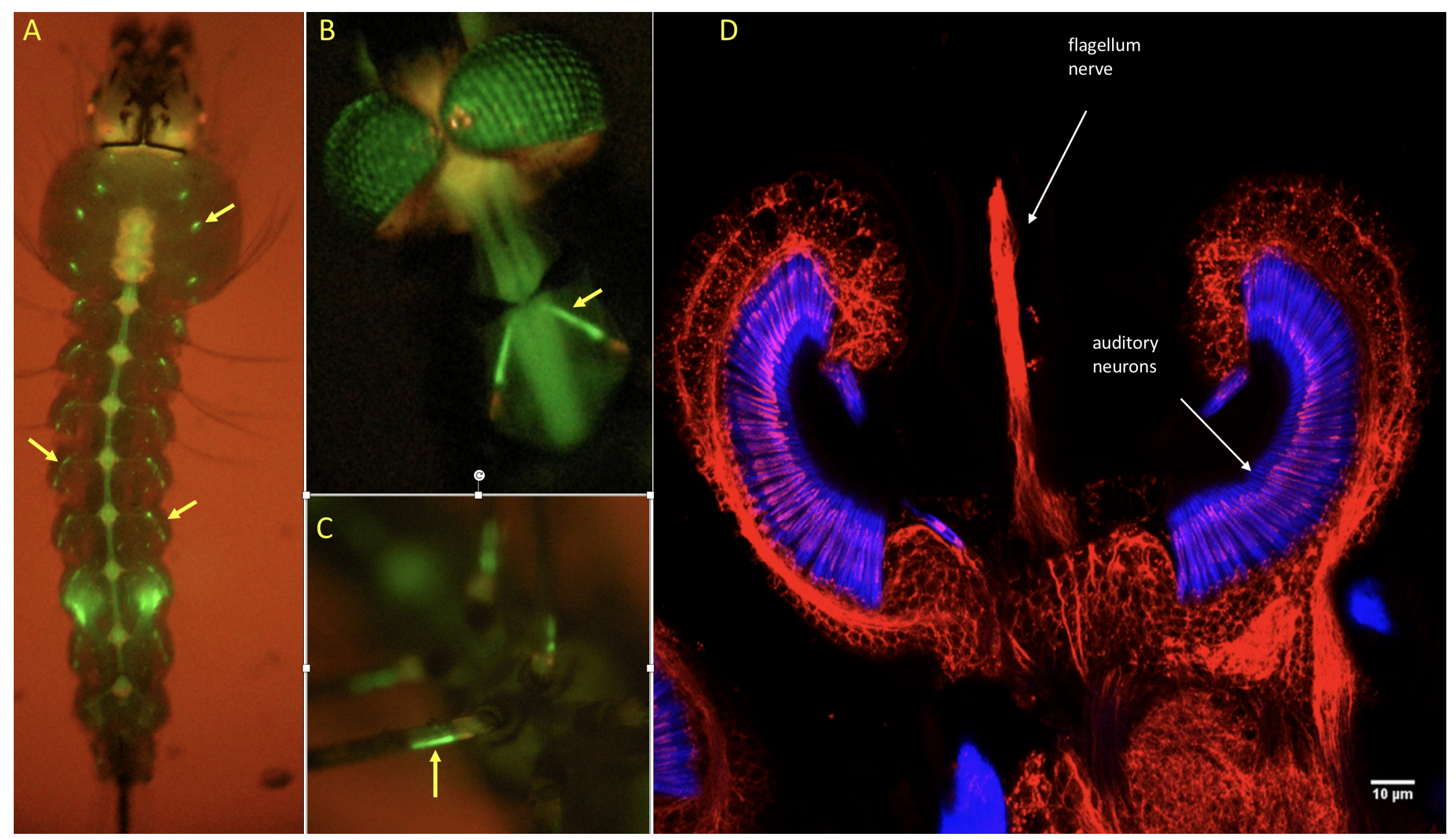


Suppression drives

The modification causes homozygous lethality

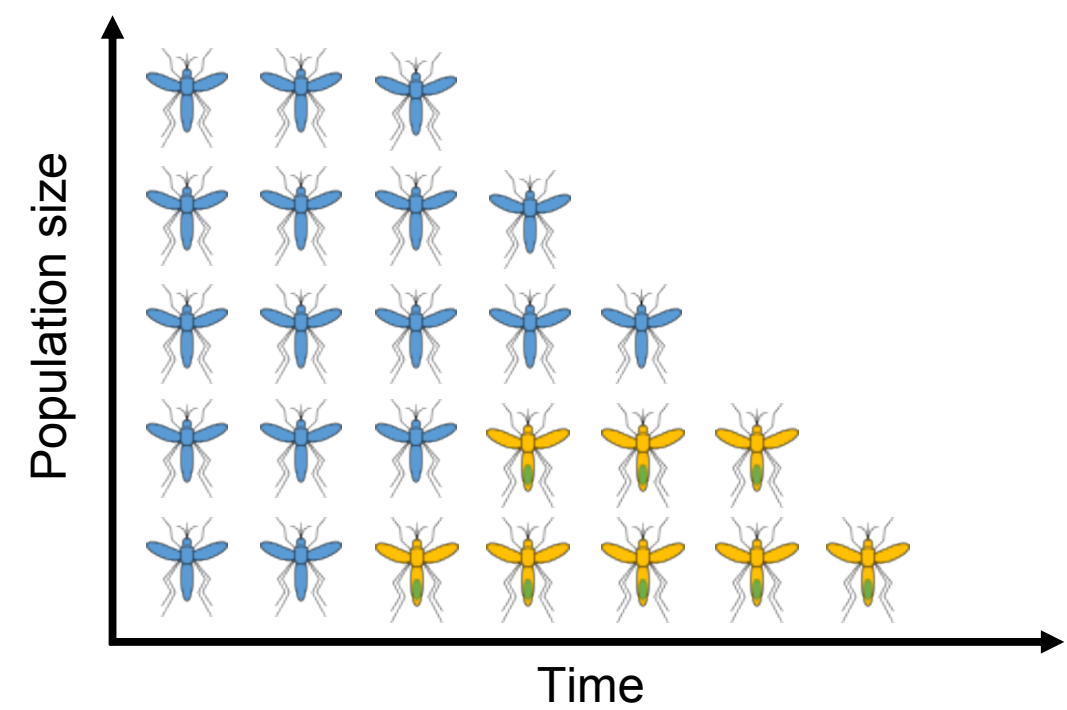

\section{Modification drives}

The modification confers refractoriness to malaria

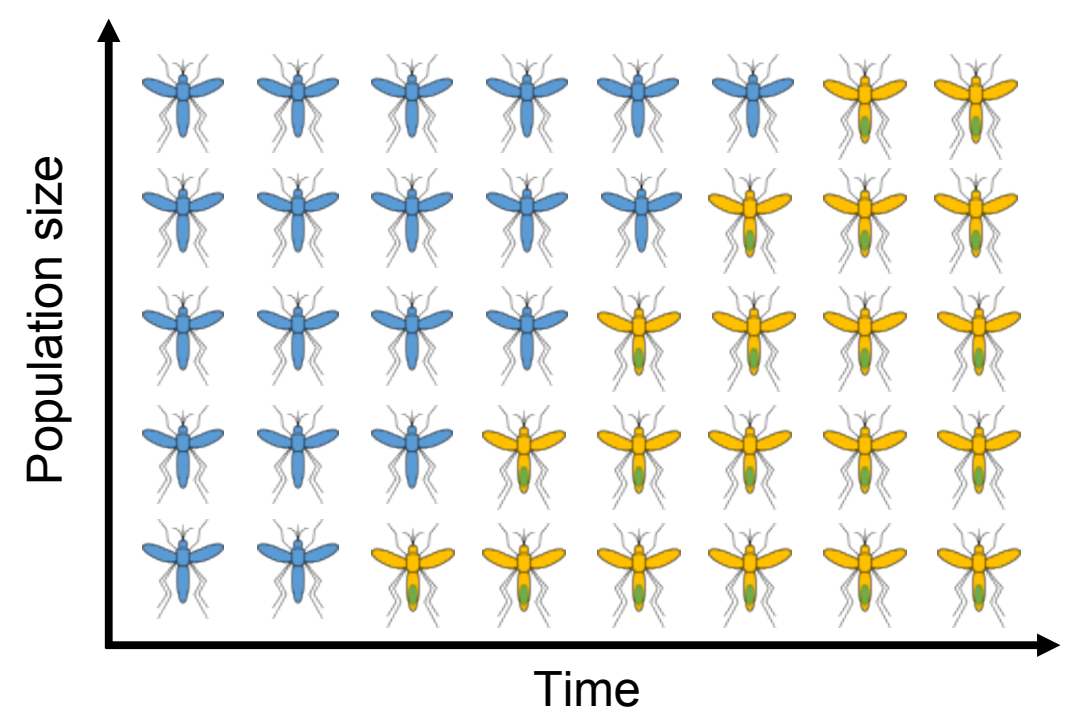

